\title{
Wichtige Schlüsselsätze für den Zahnexperten
}

\author{
Der Patient kommt als zahnmedizinischer Laie in die Praxis. Auf der anderen Seite steht der \\ Zahnarzt mit seinem Expertenteam. Er übernimmt damit auch die Verantwortung für den Laien: \\ mit zahnmedizinischer Logik und Schlüsselsätzen erklärt er dem Patienten die Behandlung. \\ Phrasendrescherei ist dabei genauso wenig angesagt wie zu vorsichtiges Nachfragen. Es muss \\ klar sein, wer der Experte ist.
}

Dabei kommt es auf die Wortwahl an. In der Variante 1 sagt der Zahnarzt zum Patienten: „Vielleicht sollten wir es mal mit der Prophylaxe bei Ihnen probieren, oder was meinen Sie?" Möglicherweise haben Sie den Satz auch schon mal benutzt? Und nun kommt Variante 2 mit identischem Inhalt, aber anders formuliert: „Ihre Zähne brauchen dringend Hilfe! Ohne Prophylaxe haben Ihre Zähne keine Chance. Deshalb sollte Sie schon möglichst in den nächsten zwei bis drei Wochen damit beginnen!“. Keine Frage, mit welchem Satz mehr Expertise ausgedrückt wird. Variante 2 ist ein gutes Beispiel für einen klassischen Expertensatz.

\section{Das Gespräch wird in unterschiedliche Bahnen gelenkt} Bei Satz Nr. 1 wird vermutlich eine lange Diskussion daraus. Denn der Patient wird sich geradezu aufgefordert fühlen, das Weichmacher-Wort „vielleicht“ zu hinterfragen. „Gibt es denn noch andere Möglichkeiten?" könnte eine Rückfrage sein, zu der durch das Fragezeichen am Ende des Satzes ja auch aufgefordert wurde. Oft folgen zeitfressende Diskussionen, und schließlich fühlt sich der Patient unwohl und weiß immer noch nicht, was er tun soll. Als Laie erwartet er das aber von dem Experten.

\section{Aussage statt Frage - ethisch vertretbar?}

Variante 2 wirkt wesentlich deutlicher, der Patient bekommt eine klare Einschätzung und einen Folgetermin. Nach diesem Folgetermin und der entsprechenden Behandlung ist er zahnmedizinisch gut aufgestellt für die Zukunft. Durch den Expertensatz hat er gar keine Alternative. Natürlich muss so eine Vorgehensweise ethisch abgeklärt sein. Gleichwohl sei die Frage erlaubt: Wie ethisch ist Variante Nr. 1? Der Patient geht ohne Behandlung und ohne klares Konzept, was er für seine Zahngesundheit tun soll, nach Hause - möglicherweise ohne Folgetermin, ohne Perspektive, ohne Prophylaxe.

\section{Expertenstatus noch verstärken}

Der entsprechende Verstärker-Satz lautet: „Ich bin der festen Überzeugung, dass...“. Dieser Satz kann zum Beispiel vorangestellt (bei Variante 2), aber auch nach einer klaren Vereinbarung genutzt werden, um den Patienten in seiner gefällten Entscheidung nochmals zu bestärken. Mit einem eindringlichen „Ich bin der festen Überzeugung, dass Sie mit Ihrem Entschluss genau die richtige Entscheidung getroffen haben" geht der Patient mit einem guten Gefühl und einer auch emotional wirkenden Bestätigung nach Hause. Ein fester Händedruck unterstützt dabei die Aussage nonverbal.

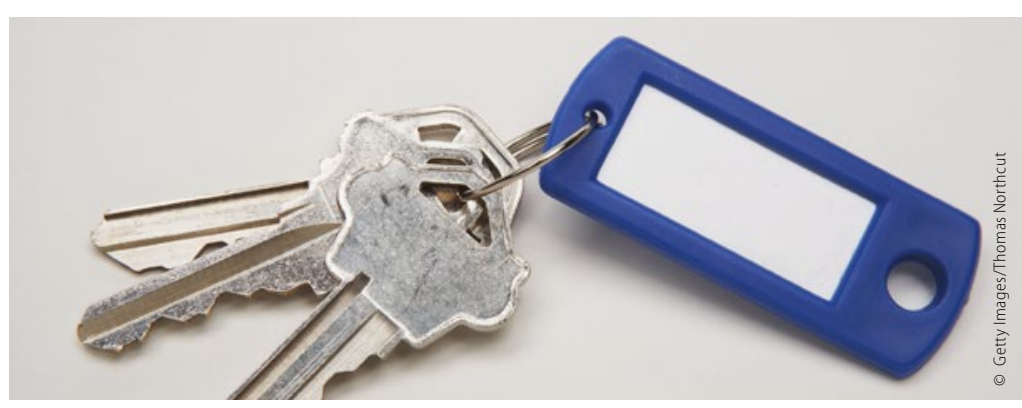

\section{Sicherheit und Vertrauen schaffen}

„Darf ich Ihnen erläutern, welche Möglichkeiten die moderne Zahnheilkunde bietet?" Bei dieser Formulierung wird der Patient ein verstecktes Verkaufsgespräch erwarten und ausweichend antworten. Die Expertenfrage mit gleichem Inhalt lautet: „Ist Ihnen schon einmal erläutert worden, welche Möglichkeiten Ihnen heute offen stehen?" Hier liegt der Fokus durch die ganz direkt Ansprache ganz eindeutig beim Wohl des Patienten. Er wird aufmerksam zuhören, denn wer möchte nicht wissen, welche Möglichkeiten es heutzutage gibt? Ist es ein neuer Patient in Ihrer Praxis, wird er sich unterbewusst Fragen: „Warum hat mir das mein letzter Arzt nicht gesagt?“ - und sich jetzt gut aufgehoben fühlen.

Der Verfasser arbeitet als Trainer, Coach und Autor im Bereich Rhetorik und Führungskommunikation. Er studierte an der Universität in Dortmund Journalistik und Organisationspsychologie. Auf seinem Internet-Blog veröffentlicht er regelmäßig Beiträge zu den Themen: Reden, Vorträge, Präsentationen mit vielen praktischen Tipps und Tricks - auch für Zahnärzte.

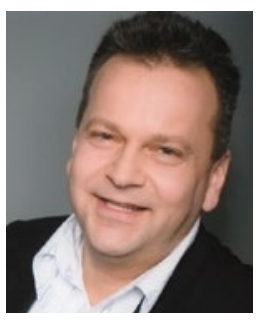

Frank Wittke

www.frank-wittke.de 